\title{
Evaluación de la inteligencia emocional y la conducta prosocial y su correlación en alumnado de Educación Primaria
}

Evaluation of emotional intelligence and prosocial behavior and their correlation among primary education students

GIADA SPORZON

Universidad de Granada gsporzon@correo.ugr.es http://orcid.org/0000-0002-6826-6847

Resumen: El estudio evalúa la inteligencia emocional y la conducta prosocial del alumnado de Educación Primaria, analiza la relación existente entre ambos constructos, y determina si hay dimensiones de la inteligencia emocional que puedan predecir la conducta prosocial. Es una investigación cuantitativa, que responde a un diseño descriptivo, correlacional y predictivo, en el que han participado 229 estudiantes. Los resultados evidencian niveles medio-altos de inteligencia emocional y conducta prosocial en el alumnado, la existencia de una relación directamente proporcional entre inteligencia emocional y conducta prosocial, y la relevancia de la dimensión interpersonal como factor predictor de la conducta prosocial.

Palabras clave: Educación Primaria, Evaluación, Inteligencia Emocional, Conducta Prosocial.
MARÍA CARMEN LóPEZ LóPEZ

Universidad de Granada

mclopez@ugr.es

https://orcid.org/0000-0002-8676-7135

Abstract: The study evaluates the emotional intelligence and prosocial behavior of primary school students; analyses the relationship between both constructs and determines whether there are dimensions of emotional intelligence that can predict prosocial behavior. It is a quantitative investigation that responds to a descriptive, correlational and predictive design, in which 229 students have participated. The results show medium-high levels of emotional intelligence and prosocial behavior in students, the existence of a directly proportional relationship between emotional intelligence and prosocial behavior, and the relevance of the interpersonal dimension as a predictor of prosocial behavior.

Keywords: Primary Education, Evaluation, Emotional Intelligence, Prosocial Behavior.

DOI: 10.15581/004.40.51-73

ESTUDIOS SOBRE EDUCACIÓN / VOL. 40 / 2021 / 51-73 


\section{INTRODUCCIÓN}

E

1 interés de los docentes e investigadores por la inteligencia emocional y la conducta prosocial del alumnado es relativamente reciente. Las razones que han contribuido a este hecho son varias y de distinta naturaleza. Junto a la necesidad de tender hacia una formación más integral que contribuya al desarrollo competencial del alumnado (Consejo de la Unión Europea, 2018) y salvar la falta de atención deparada por la escuela tradicional, que "rechaza los sentimientos y el pensamiento concreto y los aspectos de la vida humana relativos a la necesidad de vínculos emocionales" (Vázquez Verdera, 2009, p. 111), se unen las aportaciones de numerosos expertos que subrayan los beneficios de su incorporación a los procesos formativos.

La inclusión de la inteligencia emocional mejora la experiencia vital y escolar del alumnado, ya que tiene efectos positivos, entre otros aspectos, en el rendimiento académico, la adaptación social, la conducta, la autoestima, las relaciones interpersonales, la gestión del estrés, la salud física, el clima del aula y el trabajo en equipo (Vázquez de Prada y López, 2016; Domitrovich, Durlak, Staley y Weissberg, 2017; Greenberg, Domitrovich, Weissberg y Durlak, 2017). Ruvalcaba-Romero, Gallegos-Guajardo y Fuerte (2017), por ejemplo, han destacado la correlación positiva existente entre las competencias socio-emocionales, el clima escolar y la conducta prosocial. Otros autores, en cambio, han subrayado la contribución de la inteligencia emocional a la superación de las adicciones y los problemas de adaptación social (Parker, Summerfeldt, Taylor, Kloosterman y Keefer, 2013; Resurrección, Salguero y Ruiz-Aranda, 2014).

Por otro lado, la conducta prosocial reporta beneficios no sólo en la mejora de las relaciones interpersonales y de la convivencia en los microcontextos (familia, grupo de amigos, aula o escuela), sino también en los macrocontextos (colectivos sociales y políticos), como han señalado Correa (2017), Tur-Porcar, Doménech y Mestre (2018) y Barroso-Hurtado y Bembibre (2019).

Este potencial formativo de la inteligencia emocional y la conducta prosocial ha llevado a los profesionales de la educación a multiplicar, sensiblemente, el número de iniciativas educativas dirigidas a impulsar su incorporación a los procesos formativos. Las fórmulas utilizadas han sido variadas: actividades puntuales, programas educativos y, en menor medida, asignaturas específicas (Ponce y Aguaded, 2016). En el contexto internacional destacamos, por ejemplo, los programas americanos SEL, promovidos por CASEL (Weissberg, Goren, Domitrovich y Dusenbury, 2013) y, entre ellos, los programas RULER (Reyes, Brackett, Rivers, Elbertson y Salovey, 2012) y PATH (Greenberg, Kusche, Cook y Quamma, 1995), 
ambos interesados en promover la educación emocional y el desarrollo de competencias sociales como la conducta prosocial.

A nivel nacional, el interés por el desarrollo socio-emocional del alumnado se entiende vinculado a algunas de las competencias básicas y clave, incorporadas al currículo oficial por la Ley Orgánica 2/2006, de 3 de mayo, de Educación (LOE) y la Ley Orgánica 8/2013, de 9 de diciembre, para la Mejora de la Calidad Educativa (LOMCE) respectivamente. Esta conexión es especialmente relevante en las competencias que tienen un fuerte componente social y cívico. Las competencias sociales y cívicas figuran entre las competencias clave del sistema educativo español (art. 2 O. ECD/65/2015 de 21 de enero) y su propósito no es otro que el de procurar el bienestar personal y colectivo, y preparar a los estudiantes para ejercer la ciudadanía democrática y participar plenamente en la vida cívica. De esta forma, el interés educativo por la inteligencia emocional y la conducta prosocial (cooperación, empatía, solidaridad, respeto mutuo, asertividad, gestión positiva de los conflictos,...) está presente en la normativa que regula los desarrollos curriculares de las distintas etapas de la educación obligatoria.

Desde este marco general señalamos, por su relevancia, las iniciativas impulsadas por la Comunidad Autónoma de Castilla-La Mancha y la Comunidad Autónoma de Canaria. En el primer caso, la Ley 7/2010, de 20 de julio, de Educación de Castilla-La Mancha recoge, en su artículo 35 dedicado a las competencias básicas, la incorporación de la competencia emocional al currículo de los centros educativos de esta comunidad. Esta nueva competencia

se entiende como la capacidad de respuesta personal y equilibrada ante cualquier situación, la tolerancia ante la frustración y el fracaso, y el control eficaz de las consecuencias que se pueden derivar para la propia estima y para la relación con los otros (p. 86373).

En el caso de Canarias, la Consejería de Educación, Universidades y Sostenibilidad de la Comunidad, en el marco del currículo propuesto para la etapa de Educación Primaria (Decreto 89/2014), ha incluido una asignatura de libre configuración denominada Educación Emocional y para la Creatividad. Ambas incorporaciones al currículo oficial de Educación Primaria (como competencia o materia) suponen un claro respaldo de la política educativa a la inteligencia emocional, respaldo que se une a otros procedentes de otras instancias, como la Fundación Marcelino Botín, el Laboratorio de Emociones de la Universidad de Málaga y el Laboratorio de Educación Emocional de la Universidad Nacional de Educación a Distancia (Cejudo, 2015). En estos momentos, las iniciativas de educación emocional 
dirigidas a alumnado de Educación Primaria se han multiplicado de manera considerable (Melero y Palomera, 2011; Bañuls, 2015; Benito, 2017; Zorrilla, 2017). Algunas de ellas, como las impulsadas por Benito (2017) y Zorrilla (2017), abordan también las conductas prosociales. Otras, en cambio, están orientados, exclusivamente, al desarrollo de la conducta prosocial (Alba Corredor, JusticiaArráez, Pichardo y Justicia, 2013; del Moral y Villalustre, 2018). En ocasiones, la inteligencia emocional y la conducta prosocial son abordadas de manera tangencial en programas más amplios centrados en educación para la paz, educación para la convivencia o mejora de las habilidades sociales (Carrillo, 2016). Esta situación evidencia el gran interés suscitado y, al mismo tiempo, la diversidad de formatos utilizados desde los que se está abordando la inteligencia emocional y la conducta prosocial en el contexto educativo español.

Esta proliferación de iniciativas choca, no obstante, con la escasez de trabajos interesados en analizar su impacto y repercusión. Este hecho posiblemente guarda relación con la dificultad que entraña la evaluación de estos constructos (PérezEscoda, Filella, Soldevila y Fondevila, 2013). En este sentido, y junto a la dificultad que comporta la construcción de instrumentos dirigidos a la evaluación de ambos constructos, se suma la naturaleza subjetiva de las emociones (Mavroveli y Sánchez-Ruiz, 2011), la desconfianza en que éstas puedan ser evaluadas con papel y lápiz (Extremera, Fernández-Berrocal, Mestre y Guil, 2004), la escasez de instrumentos que sean válidos y fiables para el contexto español (Extremera et al., 2004) y, finalmente, y en relación a la Educación Primaria en concreto, la dificultad de diseñar instrumentos que abarquen el amplio espectro de alumnado que engloba esta etapa educativa y que se traduce en distintos grados en el dominio de la lengua, memoria, comprensión, etc. (Álvarez, Bisquerra, Fita, Martínez y Pérez, 2000). Por otra parte, la evaluación de la dimensión socio-emocional requiere una notable cantidad de tiempo (Modzelewski, 2012) que, a menudo, los profesionales de la educación reconocen no tener. Todo ello hace que existan pocos instrumentos dirigidos a la población infantil (Pérez-Escoda, Filella, Alegre y Bisquerra, 2012) y que sean igualmente escasas las experiencias formativas que se acompañan de un proceso evaluador riguroso que permita conocer el grado de desarrollo alcanzado por el alumnado en estos aspectos. Con este trabajo pretendemos salvar este vacío midiendo la inteligencia emocional y la conducta prosocial del alumnado de un colegio de Educación Primaria que tiene, en su propuesta curricular, una asignatura de inteligencia emocional.

Por otra parte, las investigaciones interesadas en estudiar la correlación entre inteligencia emocional y conducta prosocial en el contexto español son escasas, y aún menos frecuentes las enmarcadas en la etapa de Educación Primaria, ya que general- 
mente suelen focalizarse en adolescentes y Educación Secundaria. Este es el caso, por ejemplo, de los trabajos realizados por Ruvalcaba-Romero et al. (2017) y Rodríguez García (2018). En cambio, Pérez Rojo (2014) es uno de los pocos autores que ha estudiado las correlaciones entre inteligencia emocional autopercibida y conducta prosocial en alumnado de tercer ciclo de Primaria. En nuestro caso, el estudio de estas correlaciones se extiende a los tres ciclos de Educación Primaria. Se trata, pues, de una investigación novedosa en la que no sólo se evalúa la inteligencia emocional y la conducta prosocial del alumnado de los tres ciclos de Educación Primaria, sino que se clarifica y se profundiza en la relación entre ambos constructos.

\section{MATERIAL Y MÉTODO}

\section{Objetivos}

Los objetivos concretos que se persiguen en este estudio son:

1. Medir la inteligencia emocional y la conducta prosocial del alumnado de Educación Primaria.

2. Estudiar si existe relación entre la inteligencia emocional y la conducta prosocial del alumnado que participa en el estudio.

3. Determinar cuál/es de las dimensiones de la inteligencia emocional predice con mayor precisión la conducta prosocial del alumnado.

\section{Diseño}

El estudio es una investigación cuantitativa que obedece a un diseño no experimental de tipo descriptivo, correlacional y predictivo (Cohen, Manion y Morrison, 2010).

\section{Contexto y participantes}

La investigación se realiza en un colegio de Educación Primaria de la provincia de Málaga. Se trata de un centro de dos líneas, privado y bilingüe, en el que un tercio del alumnado es extranjero y de clase social media-alta. Todo el alumnado extranjero es competente en el uso de la lengua castellana para participar en el estudio y cumplimentar las escalas con garantías de fiabilidad.

Desde el curso académico 2014-2015, el colegio cuenta con una materia de libre configuración dirigida a desarrollar la inteligencia emocional de todo el alumnado. La materia se imparte durante dos horas a la semana por la psicóloga y la 
psicopedagoga del centro. Los bloques de contenido contemplados en esta materia son: inteligencia emocional y normas de cortesía, percepción emocional, comprensión emocional, autoestima, habilidades sociales y de comunicación, y habilidades para la vida y el bienestar.

Si bien la asignatura se inspira en el modelo mixto de Goleman (1995), todos los contenidos ahondan en las dimensiones de inteligencia emocional descritas por Bar-On y Parker (2000). El método seguido se apoya especialmente en la Disciplina Positiva (Nelsen, Lott y Glenn, 2008) y los materiales empleados en el aula proceden de varias fuentes, unas validadas y otras creadas ad hoc.

En el estudio han participado 229 estudiantes de Educación Primaria de un total de 238 matriculados en el curso 2017-2018. El 54.6\% de ellos son niños, el $42.4 \%$ niñas y el $3 \%$ no contesta. Todos pertenecen a los cursos de $2^{\circ}$ a $6^{\circ}$. El $20.5 \%$ son de $2^{\circ}$ curso, el $18.3 \%$ de $3^{\circ}$, el $19.2 \%$ de $4^{\circ}$ y los cursos de $5^{\circ}$ y $6^{\circ}$ están representados con el $21 \%$ cada uno.

La aplicación de las escalas se ha realizado de forma anónima durante el segundo trimestre del curso. El alumnado de $1^{\circ}$ curso de Educación Primaria no ha participado en el estudio porque los instrumentos de evaluación utilizados no los incluyen como potenciales destinatarios. En el caso de los estudiantes de $2^{\circ}$ curso la aplicación de los instrumentos se ha realizado de manera personalizada para garantizar su correcta comprensión y gracias a la ayuda de las dos profesoras que imparten la materia de inteligencia emocional.

\section{Instrumentos}

Para la recogida de información se han utilizado dos escalas: el Emotional Quotient Inventory: Young Version (EQ-i: YV), de Bar-On y Parker (2000) en su adaptación al contexto español (Ferrándiz, Hernández, Bermejo, Ferrando y Sáinz, 2012), para medir la inteligencia emocional, y la Escala de Conducta Prosocial (ECP), de Caprara y Pastorelli (1993) en su adaptación española (del Barrio, Moreno y López, 2001), para evaluar la conducta prosocial.

El primer cuestionario está formado por 60 ítems y 6 dimensiones: intrapersonal (ítems 7, 17, 28, 31, 43, 53*), interpersonal (ítems 2, 5, 10, 14, 20, 24, 36, 41, $45,51,55,59$ ), manejo del estrés (ítems $3,6^{*}, 11,15^{*}, 21^{*}, 26^{*}, 35^{*}, 39,46^{*}, 49^{*}$, $54^{*}, 58^{*}$ ), adaptabilidad (ítems 12, 16, 22, 25, 30, 34, 38, 44, 48, 57), estado de ánimo general (ítems 1, 4, 9, 13, 19, 23, 29, 32, 37*, 40, 47, 50, 56, 60) e impresión positiva (ítems $8,18,27,33,42,52)^{1}$. A partir de las 4 primeras dimensiones

1. Los ítems marcados con * son de puntuación inversa. 
se obtiene una puntuación total de inteligencia emocional, así como un cociente de inteligencia emocional a partir del producto del sumatorio promedio de dichas subescalas multiplicado por una constante $=5$. La dimensión estado de ánimo general permite conocer la situación emocional general, mientras que la impresión positiva se emplea para valorar la deseabilidad social (Ferrándiz et al., 2012). El cuestionario es una escala tipo Likert con 4 opciones de respuesta: 1 "Nunca me pasa", 2 "A veces me pasa", 3 "Casi siempre me pasa" y 4 "Siempre me pasa".

El segundo cuestionario, destinado a evaluar la conducta prosocial, se compone de 15 ítems, cinco de ellos son de control y quedarán excluidos en la presentación de los resultados. Es una escala tipo Likert con tres opciones de respuesta: "Nunca", "Algunas veces" y "A menudo".

Como parámetros de calidad de los instrumentos utilizados se han contemplado la fiabilidad y la validez. Los valores de alfa de Cronbach que determinan la fiabilidad de los instrumentos, entendida esta como consistencia interna en cada una de las dimensiones contempladas y en el total de las escalas aplicadas, han alcanzado valores que oscilan entre .60 (dimensión intrapersonal) y .81 (dimensión estado de ánimo). Para Zumbo, Gadermann y Zeisser (2007) estos valores son aceptables.

La validez de contenido de los dos instrumentos administrados se apoya en el hecho de que las dos escalas estandarizadas, como hemos señalado anteriormente, han sido validadas en el contexto español.

Finalmente, para determinar la validez criterial concurrente se ha calculado la correlación ítem-total corregida y los valores de alfa de Cronbach si se elimina el ítem en cuestión. En este caso, los valores de alfa de Cronbach, en la escala $E Q-i$ : $Y V$, oscilan entre .882 y .888 y en la ECP entre .676 y .717 . Atendiendo a los valores de correlación ítem-total corregida, las dos escalas en su conjunto presenta puntuaciones de $r> \pm 0.30$. De acuerdo con Abad, Olea, Ponsoda y García (2011), podemos afirmar que los ítems de ambas escalas miden individualmente lo que las escalas en su totalidad y, por tanto, ayudan a garantizar la unidimensionalidad de las escalas.

\section{Análisis de datos}

Los datos obtenidos tras la aplicación de los dos cuestionarios han sido analizados con el programa SPSS v. 24. Los análisis efectuados para responder a los objetivos fijados en el estudio han sido de tipo descriptivo (objetivo 1), correlacional-predictivo (objetivo 2) y de regresión lineal múltiple (objetivo 3). 


\section{RESULTADOS \\ Resultados vinculados al objetivo 1}

Para responder al objetivo 1 del estudio (Medir la inteligencia emocional y la conducta prosocial del alumnado de Educación Primaria) se han calculado las medias y desviaciones típicas de cada uno de los ítems que integran las dos escalas (EQ-i: YV y ECP). También se ha calculado el cociente emocional de la escala $E Q-i$ : $Y V$ y, finalmente, la dimensión total impresión positiva.

En la Tabla 1 presentamos las puntuaciones correspondientes a la primera de las escalas destinada a medir la inteligencia emocional.

Tabla 1. Medias y desviaciones típicas de la escala EQ-i: YV

\begin{tabular}{lll} 
ITEMS DE LA ESCALA EQ-I: $W$ & MEDIA & D.T \\
\hline 1. Me gusta divertirme & 3.67 & .617 \\
\hline 2. Entiendo bien cómo se sienten las otras personas & 2.73 & .872 \\
\hline 3. Puedo estar tranquilo cuando estoy enfadado & 2.13 & .932 \\
\hline 4. Soy feliz & 3.51 & .653 \\
\hline 5. Me importa lo que le sucede a otras personas & 3.15 & .990 \\
\hline 6. Me resulta dif́́cil controlar mi ira & 2.74 & .955 \\
\hline 7. Me resulta fácil decirle a la gente cómo me siento & 2.47 & 1.033 \\
\hline 8. Me gusta cada persona que conozco & 2.98 & .932 \\
\hline 9. Me siento seguro de mí mismo & 3.38 & .795 \\
\hline 10. Sé cómo se sienten las otras personas & 2.73 & .981 \\
\hline 11. Sé cómo mantenerme tranquilo & 2.90 & .902 \\
\hline 12. Cuando me hacen preguntas dif́́ciles, trato de responder de distintas formas & 2.68 & .995 \\
\hline 13. Pienso que la mayoría de las cosas que hago saldrán bien & 3.03 & .873 \\
\hline 14. Soy capaz de respetar a los demás & 3.43 & .707 \\
\hline 15. Algunas cosas me enfadan mucho & 2.22 & .934 \\
\hline 16. Es fácil para mi entender cosas nuevas & 2.92 & .905 \\
\hline 17. Puedo hablar con facilidad acerca de mis sentimientos & 2.49 & .987 \\
\hline 18. Tengo buenos pensamientos acerca de todas las personas & 3.03 & .821 \\
\hline 19. Espero lo mejor & 3.59 & .748 \\
\hline 20. Tener amigos es importante & 3.76 & .612 \\
\hline
\end{tabular}




\section{EVALUACIÓN DE LA INTELIGENCIA EMOCIONAL Y LA CONDUCTA PROSOCIAL}

\section{Tabla 1. Medias y desviaciones típicas de la escala EQ-i: YV}

\begin{tabular}{|c|c|c|}
\hline ITTEMS DE LA ESCALA EQ-I: W & MEDIA & D.T \\
\hline 21. Me peleo con la gente & 3.07 & .881 \\
\hline 22. Puedo entender preguntas difíciles & 2.77 & .879 \\
\hline 23. Me gusta sonreír & 3.57 & .773 \\
\hline 24. Trato de no herir los sentimientos de los otros & 3.20 & 1.045 \\
\hline 25. Trato de trabajar en un problema hasta que lo resuelvo & 3.24 & .849 \\
\hline 26. Tengo mal genio & 3.19 & .856 \\
\hline 27. Nada me molesta & 2.20 & .778 \\
\hline 28. Me resulta difícil hablar de mis sentimientos & 2.36 & 1.074 \\
\hline 29. Sé que las cosas saldrán bien & 3.13 & .825 \\
\hline 30. Ante preguntas difíciles, puedo dar buenas respuestas & 2.98 & .822 \\
\hline 31. Puedo describir mis sentimientos con facilidad & 2.43 & .996 \\
\hline 32. Sé cómo pasar un buen momento & 3.55 & .734 \\
\hline 33. Debo decir la verdad & 3.42 & .816 \\
\hline 34. Cuando quiero puedo encontrar muchas formas de contestar a una pregunta difícil & 2.94 & .933 \\
\hline 35. Me enfado con facilidad & 2.93 & .982 \\
\hline 36. Me gusta hacer cosas para los demás & 3.14 & .909 \\
\hline 37. Me siento poco feliz & 3.33 & .890 \\
\hline 38. Puedo resolver problemas de diferentes maneras & 3.01 & .906 \\
\hline 39. Tienen que pasarme muchas cosas para que me enfade & 2.50 & 1.011 \\
\hline 40. Me siento bien conmigo mismo & 3.49 & .776 \\
\hline 41. Hago amigos con facilidad & 3.29 & .907 \\
\hline 42. Pienso que soy el mejor en todo lo que hago & 2.00 & 1.043 \\
\hline 43. Es fácil para mí decirle a la gente lo que siento & 2.34 & 1.021 \\
\hline 44. Cuando contesto preguntas difíciles, trato de pensar en muchas soluciones & 3.02 & .915 \\
\hline 45. Me siento mal cuando se hieren los sentimientos de otras personas & 3.09 & .953 \\
\hline 46. Cuando me enfado con alguien, me enfado durante mucho tiempo & 2.93 & .917 \\
\hline 47. Soy feliz con el tipo de persona que soy & 3.57 & .773 \\
\hline 48. Soy bueno para resolver problemas & 3.05 & .839 \\
\hline 49. Me resulta difícil esperar mi turno & 2.76 & .994 \\
\hline 50. Me entretienen las cosas que hago & 3.22 & .896 \\
\hline
\end{tabular}

[CONTINÚA EN LA PÁGINA SIGUIENTE] 
Tabla 1. Medias y desviaciones típicas de la escala EQ-i: YV

\begin{tabular}{lcc} 
ITEMS DE LA ESCALA EQ-I: $W$ & MEDIA & D.T \\
\hline 51. Me gustan mis amigos & 3.68 & .655 \\
\hline 52. No tengo días malos & 2.45 & .845 \\
\hline 53. Tengo problemas para hablar de mis sentimientos a los demás & 2.49 & 1.099 \\
\hline 54. Me enfado con facilidad & 2.93 & .968 \\
\hline 55. Puedo darme cuenta cuando uno de mis mejores amigos no es feliz & 3.36 & .866 \\
\hline 56. Me gusta mi cuerpo & 3.41 & .867 \\
\hline 57. Aun cuando las cosas se ponen difíciles, no me doy por vencido & 3.08 & .966 \\
\hline 58. Cuando me enfado, actúo sin pensar & 2.52 & 1.015 \\
\hline 59. Sé cúando la gente está enfadada, incluso cuando no dicen nada & 2.98 & .962 \\
\hline 60. Me gusta cómo me veo & 3.46 & .79 \\
\hline
\end{tabular}

El promedio total de la escala $E Q-i$ : $Y V$ es 2.9. Las medias más altas, por dimensiones, son estado de ánimo (3.41) y dimensión interpersonal (3.21), mientras que las puntuaciones más bajas se encuentran en manejo del estrés (2.73) y dimensión intrapersonal (2.43).

Cabe destacar que, en su mayor parte, las puntuaciones típicas más altas corresponden a ítems pertenecientes a la subescala que estudia las habilidades intrapersonales, concretamente en: "Tengo problemas para hablar de mis sentimientos a los demás" (ítem 53, s=1.099), "Me resulta fácil decirle a la gente cómo me siento" (ítem 7, s= 1.074), "Me resulta difícil hablar de mis sentimientos" (ítem 28, $s=1.074)$ y "Es fácil para mí decirle a la gente lo que siento" (ítem 43, s=1.021).

El valor del cociente emocional de la escala $E Q-i$ : $Y V$ es 56.69. Finalmente, en cuanto a la dimensión impresión positiva, los resultados ponen en evidencia que el alumnado no presenta el sesgo de la deseabilidad social (coeficiente de asimetría $=-.04)$.

En la Tabla 2 mostramos los valores medios y las desviaciones típicas obtenidos en la escala relativa a la conducta prosocial.

Tabla 2. Medias y desviaciones típicas de la escala ECP

\begin{tabular}{llc} 
ÍTEMS DE LA ESCALA ECP & MEDIA & D. T. \\
\hline 1. Intento consolar al que está triste & 2.55 & .557 \\
\hline 2. Hago compañía a mis amigos/as & 2.82 & .396 \\
\hline 3. Intento ayudar a los demás & 2.73 & .484 \\
\hline
\end{tabular}


Tabla 2. Medias y desviaciones típicas de la escala ECP

\begin{tabular}{llc} 
ITEMS DE LA ESCALA ECP & MEDIA & D. T. \\
\hline 4. Soy amable & 2.69 & .518 \\
\hline 5. Comparto con los/las amigos/as las cosas que me gustan & 2.63 & .536 \\
\hline 6. Ayudo a mis compañeros/as a hacer sus deberes & 2.74 & .468 \\
\hline 7. Presto mis juegos & 2.48 & .633 \\
\hline 8. Me gusta jugar en compañía & 2.71 & .526 \\
\hline 9. Confío en los demás & 2.46 & .534 \\
\hline 10. Soy cariñoso/a con mis amigos/as & 2.39 & .729 \\
\hline
\end{tabular}

Con respecto a la escala $E C P$, el promedio total es de 2.62. Los ítems mejor valorados son el 2 "Hago compañía a mis amigos" (2.82), el 6 "Ayudo a mis compañeros/ as a hacer sus deberes" (2.74) y 3 "Intento ayudar a los demás" (2.73), mientras que las medias más bajas corresponden a los ítems 10 "Soy cariñoso/a con mis amigos/ as" (2.39), 9 "Confío en los demás" (2.46) y 7 "Presto mis juegos" (2.48). Cabe destacar la heterogeneidad en las respuestas de los alumnos, sobre todo en el ítem 10 "Soy cariñoso/a con mis amigos/as" ( $s=.729)$.

\section{Resultados vinculados al objetivo 2}

En este apartado se presentan los resultados obtenidos tras el análisis correlacionalpredictivo de la escala de inteligencia emocional, y sus diferentes componentes, con la escala de conducta prosocial. A continuación se muestra el diagrama de dispersión matricial y la matriz de correlaciones de las dimensiones de inteligencia emocional que conforman la escala $E Q-i$ : $Y V$, más el cociente inteligencia emocional y el total de dicha escala, así como el total de escala de conducta prosocial. No obstante, las correlaciones que más interesan para responder al objetivo 2 sólo son aquellas que se establecen entre el total de la escala de conducta prosocial con las dimensiones interpersonal, intrapersonal, manejo del estrés y adaptabilidad de la escala $E Q-i: Y V$, más el cociente de inteligencia emocional y el total de dicha escala. Destacamos, por tanto, estas correlaciones. 
Gráfico 1. Dispersión matricial de las dimensiones de la escala EQ-i: YV, el cociente emocional, el total de escala EQ-i: YV y el total de escala prosocial

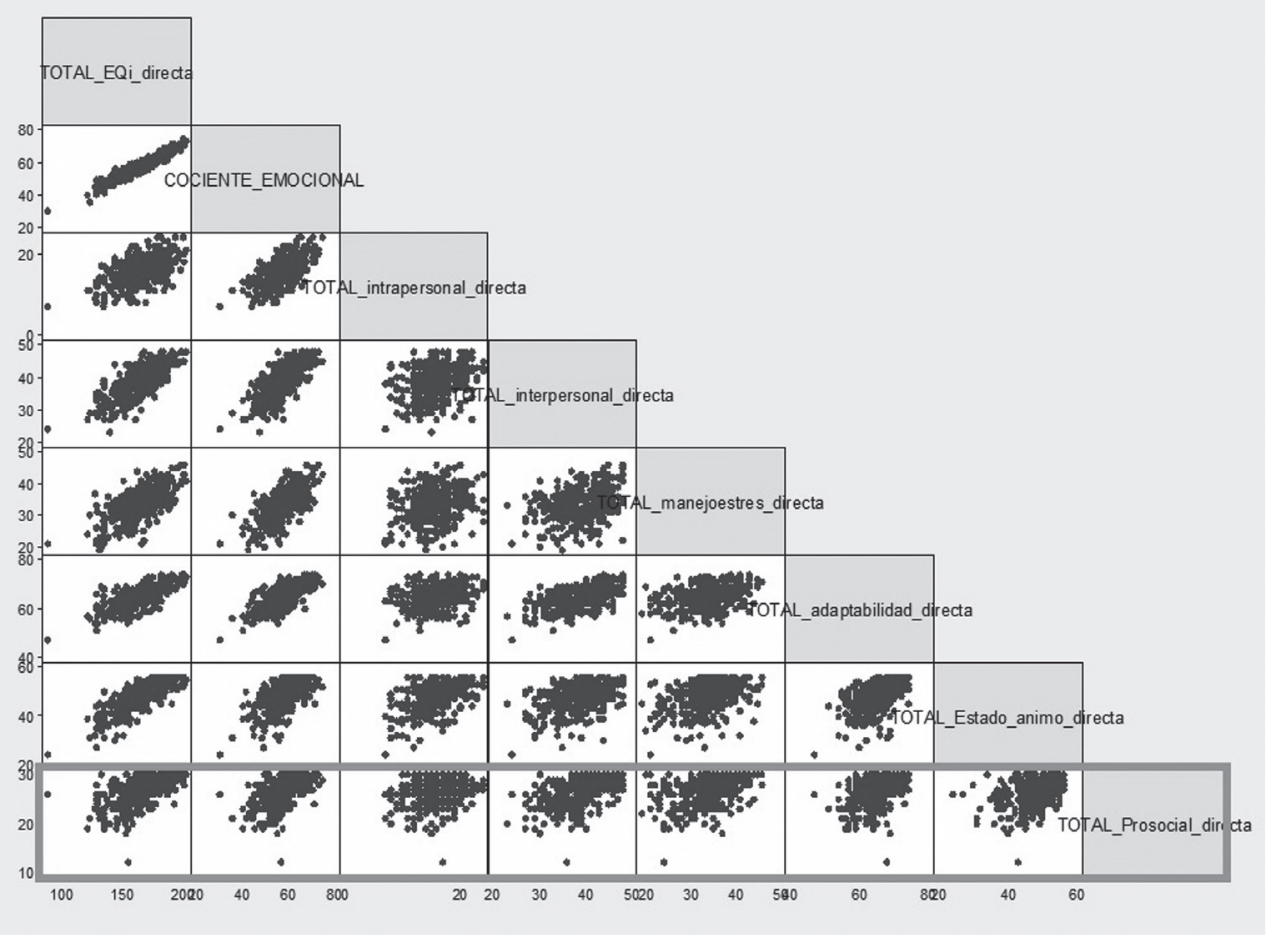

Como recoge el Gráfico 1 de dispersión matricial y la matriz de correlaciones de la Tabla 3, el alumnado muestra correlaciones de Pearson que median entre los valores $\mathrm{r}=.23$ (con la dimensión intrapersonal) y $\mathrm{r}=.56$ (con el total de la escala de inteligencia emocional), todas con signo positivo (obviando, lógicamente, la correlación de la conducta prosocial consigo misma, cuya correlación es perfecta, es decir, $r=1)$.

Lo más relevante es que todas las correlaciones, independientemente de su importancia, son estadísticamente significativas ( $\mathrm{p}=.000$ en todos los casos). Esto indica que todas las dimensiones correlacionadas con la conducta prosocial guardan una relación directamente proporcional, es decir, que cuando aumenta una variable (cualquiera de las contempladas) correlacionada con conducta prosocial, también aumenta ésta y viceversa. Otro dato relevante es que las relaciones que se establecen no se deben al azar. 
Tabla 3. Matriz de correlaciones de las dimensiones de la escala EQ-i: YV, el cociente emocional, el total de escala EQ-i: YV y total de escala prosocial

\begin{tabular}{|c|c|c|c|c|c|c|c|c|}
\hline & & $\begin{array}{l}\text { TOTAL_Proso } \\
\text { cial_directa }\end{array}$ & $\begin{array}{l}\text { TOTAL_intrap } \\
\text { ersonal_direc } \\
\text { ta }\end{array}$ & $\begin{array}{l}\text { TOTAL_interp } \\
\text { ersonal_direc } \\
\text { ta }\end{array}$ & $\begin{array}{l}\text { TOTAL_mane } \\
\text { joestres_dire } \\
\text { cta }\end{array}$ & $\begin{array}{l}\text { TOTAL_adapt } \\
\text { abilidad_dire } \\
\text { cta }\end{array}$ & $\begin{array}{l}\text { TOTAL_Estad } \\
\text { o_animo_dire } \\
\text { cta }\end{array}$ & $\begin{array}{l}\text { TOTAL_EQi__ } \\
\text { directa }\end{array}$ \\
\hline \multirow[t]{3}{*}{ TOTAL_Prosocial_directa } & Correlacion de Pearson & 1 &, 233 & .527 &, 388 &, 396 &, 458 &, 564 \\
\hline & Sig. (bilateral) & &, 000 &, 000 & .000 &, 000 &, 000 &, 000 \\
\hline & $\mathrm{N}$ & 228 & 228 & 228 & 228 & 228 & 228 & 228 \\
\hline \multirow{3}{*}{$\begin{array}{l}\text { TOIAL_Intrapersonal_dir } \\
\text { ecta }\end{array}$} & Correlacion de Pearson & .233 & 1 & .250 & .256 & .236 & .479 & .561 \\
\hline & Sig. (bilateral) &, 000 & &, 000 & .000 & .000 & .000 &, 000 \\
\hline & $\mathrm{N}$ & 228 & 229 & 229 & 229 & 229 & 229 & 229 \\
\hline \multirow{3}{*}{$\begin{array}{l}\text { TOTAL_interpersonal_dir } \\
\text { ecta }\end{array}$} & Correlación de Pearson &, $527^{11}$ & $.250^{\circ \prime}$ & 1 & $.344^{\prime \prime}$ &, $591^{\prime \prime}$ & $.536^{\prime \prime}$ &, $773^{11}$ \\
\hline & Sig. (bilateral) &, 000 & .000 & & .000 & .000 & .000 & .000 \\
\hline & $\mathrm{N}$ & 228 & 229 & 229 & 229 & 229 & 229 & 229 \\
\hline \multirow{3}{*}{$\begin{array}{l}\text { TOTAL_manejoestres_di } \\
\text { recta }\end{array}$} & Correlación de Pearson &, $388^{\prime \prime \prime}$ & $.256^{\prime \prime}$ & $.344^{\prime \prime}$ & 1 & $.293^{\prime \prime \prime}$ &, $355^{\prime \prime}$ & $.661^{\prime \prime}$ \\
\hline & Sig. (bilateral) & .000 & .000 &, 000 & & .000 & .000 &, 000 \\
\hline & $\mathrm{N}$ & 228 & 229 & 229 & 229 & 229 & 229 & 229 \\
\hline \multirow{3}{*}{$\begin{array}{l}\text { TOTAL_adaptabilidad_dir } \\
\text { ecta }\end{array}$} & Correlación de Pearson &, $396^{\prime \prime}$ & $.236^{\prime \prime}$ & $.591^{\prime \prime}$ & $.293^{\prime \prime \prime}$ & 1 & $.544^{\prime \prime}$ & $.733^{11}$ \\
\hline & Sig. (bilateral) & .000 & .000 & .000 & .000 & & .000 &, 000 \\
\hline & $\mathrm{N}$ & 228 & 229 & 229 & 229 & 229 & 229 & 229 \\
\hline \multirow{3}{*}{$\begin{array}{l}\text { TOTAL_Estado_animo_d } \\
\text { irecta }\end{array}$} & Correlación de Pearson & $.458^{\prime \prime}$ & $.479^{\prime \prime}$ & $.536^{\prime \prime}$ & $.355^{112}$ & $.544^{\prime \prime}$ & 1 & $.811^{11}$ \\
\hline & Sig. (bilateral) & .000 & .000 &, 000 & .000 & .000 & & .000 \\
\hline & $\mathrm{N}$ & 228 & 229 & 229 & 229 & 229 & 229 & 229 \\
\hline \multirow[t]{3}{*}{ TOTAL_EQi_directa } & Correlación de Pearson &, $564^{\prime \prime}$ &, $561^{\prime \prime}$ & $.773^{\prime \prime}$ & $.661^{\prime \prime}$ &, $733^{\prime \prime}$ & $811^{\prime \prime}$ & 1 \\
\hline & Sig. (bilateral) &, 000 & .000 &, 000 & .000 & .000 & .000 & \\
\hline & $\mathrm{N}$ & 228 & 229 & 229 & 229 & 229 & 229 & 229 \\
\hline
\end{tabular}

** La correlación es significativa en el nivel .01 (2 colas).

\section{Resultados vinculados al objetivo 3}

Para determinar la dimensión o dimensiones de la inteligencia emocional que mejor predicen la conducta prosocial del alumnado se ha realizado un análisis de regresión lineal múltiple en el que el total de la prueba de conducta prosocial ha actuado como variable criterio o dependiente. El resto de dimensiones anteriormente correlacionadas con ella (interpersonal, intrapersonal, manejo del estrés, adaptabilidad y estado de ánimo), más el cociente emocional y el total de dicha escala han cumplido el rol de predictores. Todos ellos han sido introducidos según la magnitud de correlación, de mayor a menor, que guardan con la conducta prosocial.

En la Tabla 4 se muestran la/s variable/s introducidas/eliminadas en cada modelo tras la aplicación del método de pasos sucesivos (stepwise) y los criterios: probabilidad-de-F-para-entrar $<=.050$, probabilidad-de-F-para-eliminar $>=.100$. 
Tabla 4. Variable-s introducida-s/eliminada-s en los modelos de regresión inferidos

\begin{tabular}{|c|c|c|c|}
\hline MODELO & $\begin{array}{l}\text { VARIABLES } \\
\text { INTRODUCIDAS }\end{array}$ & $\begin{array}{l}\text { VARIABLES } \\
\text { ELIMINADAS }\end{array}$ & MÉTODO \\
\hline 1 & TOTAL_EQi_directa & el resto de predictores & $\begin{array}{l}\text { Por pasos (Criterios: Probabilidad-de-F-para- } \\
\text { entrar }<=.050, \text { Probabilidad-de-F-para- } \\
\text { eliminar }>=.100)\end{array}$ \\
\hline 2 & $\begin{array}{l}\text { TOTAL_EQi_directa + } \\
\text { TOTAL_interpersonal__ } \\
\text { directa }\end{array}$ & $\begin{array}{l}\text { el resto de predictores } \\
\text { menos EQi directa }\end{array}$ & $\begin{array}{l}\text { Por pasos (Criterios: Probabilidad-de-F-para- } \\
\text { entrar }<=.050, \text { Probabilidad-de-F-para- } \\
\text { eliminar }>=.100)\end{array}$ \\
\hline
\end{tabular}

a. Variable dependiente: TOTAL_Prosocial_directa.

Como se puede apreciar, el método de pasos sucesivos ha inferido 2 modelos, y en cada uno de ellos ha introducido un predictor. Como es un método de pasos sucesivos, el modelo definitivo es el modelo 2, por lo tanto se descarta el modelo 1. En el modelo 2 (definitivo), de todos los predictores contemplados, sólo han sido seleccionados el total del EQi y la dimensión interpersonal de la inteligencia emocional; se han eliminado el resto de predictores.

A continuación mostramos el resumen del modelo 2 (Tabla 5), donde se muestra el coeficiente de correlación múltiple obtenido, así como los coeficientes de correlación cuadrático y cuadrático ajustado, también llamados coeficientes de determinación, y el error estándar de la estimación. Estos estadísticos permiten valorar la precisión predictiva del modelo inferido.

Tabla 5. Resumen del modelo

\begin{tabular}{lcccc} 
MODELO & $\mathrm{R}$ & RCUADRADO & RCUADRADO AJUSTADO & ERROR ESTÁNDAR \\
\hline 1 & $.564^{\mathrm{a}}$ & .318 & .315 & 2.42 \\
\hline 2 & $.582^{\mathrm{b}}$ & .339 & .333 & 2.39 \\
\hline
\end{tabular}

a. Predictores: (Constante), TOTAL_EQi_directa.

b. Predictores: (Constante), TOTAL_EQi_directa, TOTAL_interpersonal_directa.

De esta forma, constatamos que el coeficiente de correlación múltiple asciende a $\mathrm{r}=.582$, un valor moderadamente alto asociado a unos coeficientes de determinación que ascienden a $\mathrm{R}^{2}=.339 \mathrm{y} \mathrm{R}^{2}{ }_{\text {ajustado }}=.333$. Una interpretación plausible del coeficiente de determinación ajustado es que el 33.3\% de la variabilidad de la conducta prosocial es explicada por el modelo de regresión conformado por las variables inteligencia emocional (total prueba) y total dimensión interpersonal. 
Otro indicador del buen ajuste del modelo es la obtención de un error típico de estimación See=2.39, que puede considerarse bajo. Teniendo en cuenta que este error se basa en las diferencias entre las puntuaciones reales del criterio $(y)$ y las que predicen el modelo $\left(y^{\wedge}\right)$, podemos considerar que el error es bajo y que, por tanto, las puntuaciones reales del criterio se parecen a las que predice el modelo.

Finalmente, en la Tabla 6 se presentan los coeficientes de la ecuación de regresión correspondientes al modelo 2 . Todos los coeficientes resultantes, tanto "a" $o$ constante como las pendientes o tangentes $b_{\text {1total EQi }} \mathrm{y}_{\text {2interpesonal }}$ (las dos positivas), están asociados a niveles de probabilidad $\mathrm{p}=.000$, es decir, estadísticamente significativos y, por tanto, deben formar parte de la ecuación de regresión.

\section{Tabla 6. Coeficientes de la ecuación de regresión resultante correspondientes al modelo 2}

\begin{tabular}{|c|c|c|c|c|c|c|}
\hline & & $\begin{array}{r}\text { CO } \\
\text { NOEST }\end{array}$ & $\begin{array}{l}\text { JTES } \\
\text { RIZADOS }\end{array}$ & $\begin{array}{c}\text { COEFICIENTES } \\
\text { ESTANDARIZADOS } \\
\end{array}$ & & \\
\hline & & B & $\begin{array}{c}\text { ERROR } \\
\text { ESTÁNDAR }\end{array}$ & BETA & $\mathrm{T}$ & SIG. \\
\hline 1 & (Constante) & 11.030 & 1.486 & & 7.421 & $.000 * * *$ \\
\hline & TOTAL_EQi_directa & .093 & .009 & .564 & 10.271 & $.000 * * *$ \\
\hline 2 & (Constante) & 10,886 & 1.468 & & 7.416 & $.000 * * *$ \\
\hline & TOTAL_EQi_directa & .064 & .014 & .389 & 4.553 & $.000 * * *$ \\
\hline & $\begin{array}{l}\text { TOTAL_interpersonal_ } \\
\text { directa }\end{array}$ & .126 & .047 & .227 & 2.652 & $.009 * * *$ \\
\hline
\end{tabular}

Probabilidades "p" estadísticamente significativas: * $p<.05 * * p<.01 * * * p<.001$.

La ecuación de regresión lineal múltiple resultante en notación estadística es la siguiente:

$$
\begin{aligned}
& \mathrm{Y}^{\wedge}=a+b_{1} x_{1}+b_{2} x_{2} \\
& \mathrm{Y}^{\wedge}{ }_{\text {conducta prosocial }}=11.030+.064 x_{1}+.126 x_{2}
\end{aligned}
$$

Según la ecuación de regresión lineal múltiple resultante, la conducta prosocial del alumnado está estrechamente influida por la inteligencia emocional en su totalidad y por la dimensión interpersonal de dicha inteligencia. Cuando la inteligencia emocional, en su totalidad, aumenta una unidad (predictor $\mathrm{b}_{1}$ ), la conducta prosocial aumenta en .064 unidades, y cuando es la dimensión interpersonal de la inteligencia emocional la que aumenta una unidad (predictor $\mathrm{b}_{2}$ ), la conducta prosocial aumenta en .126 unidades. 


\section{DisCUSIÓN}

Los resultados obtenidos revelan que los niveles de inteligencia emocional y conducta prosocial del alumnado que ha participado en la investigación son medio-altos. Estos datos positivos han sido confirmados en otros estudios que han analizado la repercusión de las iniciativas de educación emocional en los estudiantes (FilellaGuiu, Pérez-Escoda, Agulló y Oriol, 2014; Tur-Porcar, Mestre, Samper, Malonda y Llorca, 2014; Vázquez de Prada y López, 2016; Viguer, Cantero y Bañuls, 2017). Algunos de ellos, como el realizado por Tur-Porcar et al. (2014), responden a investigaciones cuasi-experimentales, con pretest y postest, lo que permite vincular las puntuaciónes alcanzadas por el alumnado con las actuaciones emprendidas para mejorar su inteligencia emocional. En nuestro caso, en cambio, dado que todo el alumnado de Educación Primaria que participa en el estudio cursa la materia de educación emocional, no es posible afirmar, de manera categórica, que los resultados positivos obtenidos son consecuencia de las actuaciones emprendidas por el profesorado en la materia de inteligencia emocional. Tampoco estamos en disposición de rechazar esa posibilidad. Cabe la opción, no obstante, de replicar el estudio y ampliar su fiabilidad incrementando el tamaño de la muestra.

En este sentido, sería igualmente interesante la posibilidad de emprender un estudio longitudinal en el colegio analizado que permitiera conocer la evolución experimentada, a nivel de resultados, en los distintos ciclos de Educación Primaria a medida que aumentan los años en que se cursa la materia, así como el grado de sostenibilidad de los logros ya alcanzados y recogidos en este estudio, tal y como sugieren Palomera, Melero y Briones (2018) y Panayiotou, Humphrey y Wigelsworth (2019). Esta opción podría ser un buen detonante para impulsar procesos de innovación y mejora permanentes en las escuelas dirigidos al desarrollo de las competencias socio-emocionales y la conducta prosocial del alumnado. Existen, en este sentido, algunos referentes experienciales exitosos, como las iniciativas presentadas por Weissberg et al. (2013), Crean y Johnson (2013) y Reyes et al. (2012).

Los resultados obtenidos confirman, igualmente, que la mejora de la inteligencia emocional aumenta la prosocialidad del alumnado, como han señalado Ruvalcaba-Romero et al. (2017); Blewitt et al. (2018) y Guo, Sun, Cai, Zhang y Song (2019), y viceversa (Aknin, van de Vondervoort y Hamlin, 2018; Tur-Porcar et al., 2018). De esta forma, se ratifica la correlación positiva existente entre ambos constructos y señalada en el estudio descriptivo realizado por Pérez Rojo (2014), con las mismas escalas y alumnado de Educación Primaria, y la investigación llevada a cabo por Rodríguez (2018) con estudiantes de Educación Secundaria. En el caso concreto del alumnado que participa en esta investigación, el hecho de que 
la conducta prosocial no forme parte del contenido contemplado explícitamente en la asignatura de inteligencia emocional, nos lleva a pensar que los buenos resultados obtenidos en este constructo podrían ser consecuencia de los progresos experimentados en inteligencia emocional. Esta apreciación adquiere valor cuando se confirma, según los resultados de esta investigación, que existe una relación directamente proporcional entre la conducta prosocial y la inteligencia emocional.

Por otra parte, el estudio no sólo clarifica la correlación existente entre conducta prosocial e inteligencia emocional y su proporcionalidad, sino que evidencia que el elemento que mejor predice la conducta prosocial, aparte de la inteligencia emocional, es, especialmente, la dimensión interpersonal de la misma, como también ha señalado Kusumaningrum (2019) en un estudio reciente. Esto implica que aspectos como la empatía, la responsabilidad social y la habilidad para establecer relaciones interpersonales son elementos clave en el desarrollo de la conducta prosocial del alumnado de Educación Primaria. Esta consideración, analizada desde una perspectiva preventiva, puede ser de gran interés para prevenir las conductas disruptivas del alumnado en etapas educativas posteriores (Caprara et al., 2015; Rodríguez García, 2018).

Consideramos, así mismo, que la evaluación de la inteligencia emocional y la conducta prosocial, además de apoyarse en los datos cuantitativos proporcionados por los estudiantes, debería contemplar también sus vivencias y percepciones desde un enfoque más cualitativo (Coskun, 2019) e incorporar, también, la información proporcionada por el profesorado y las familias, como sugieren Bisquerra, Martínez, Obiols y Pérez (2006) y Melero y Palomera (2011).

Finalmente, aunque valoramos positivamente, al igual que Pulido y Herrera (2017), las iniciativas dirigidas a favorecer la inteligencia emocional del alumnado en Educación Primaria, consideramos que su abordaje curricular, además de disciplinar, ha de tener un carácter transversal. Esto ayudaría a impregnar el conjunto de experiencias educativas que los niños desarrollan en el marco escolar más allá de programas y materias concretas (Lemes, García y García, 2014; Hortigüela, Ausín, Abella y Delgado, 2017). Avanzar en esta dirección requiere de compromisos colectivos y explícitos que comprometen a la política educativa, a la comunidad educativa y, de manera especial, a los equipos directivos (Cefai, Bartolo, Cavioni y Downes, 2018) y al profesorado en su conjunto (Fuster y Altamirano, 2017).

Fecha de recepción del original: 25 de junio 2020

Fecha de aceptación de la versión definitiva: 15 de septiembre 2020 


\section{REFERENCIAS}

Abad, F., Olea, J., Ponsoda, V. y García, C. (2011). Medición en ciencias sociales y de la salud. Madrid: Síntesis.

Aknin, L. B., van de Vondervoort, J. W. y Hamlin, J. K. (2018). Positive feelings reward and promote prosocial behavior. Current Opinion in Psychology, 20, 5559. https://doi.org/10.1016/j.copsyc.2017.08.017.

Alba Corredor, G., Justicia-Arráez, A., Pichardo, M. C. y Justicia, F. (2013). Aprender a Convivir. Un programa para la mejora de la competencia social del alumnado de Educación Infantil y Primaria. Electronic Journal of Research in Educational Psychology, 11(3), 883-904. https://doi.org/10.14204/ejrep.31.13105

Álvarez, M., Bisquerra, R., Fita, E., Martínez, F. y Pérez, N. (2000). Evaluación de programas de educación emocional. Revista de Investigación Educativa, 18(2), 587-599. https://revistas.um.es/rie/article/view/121241

Bañuls, R. (2015). Promoviendo la inteligencia emocional en la escuela: diseño y evaluación del programa EDI (Tesis doctoral, Universidad de Valencia).

Bar-On, R. y Parker, J. D. A. (2000). EQ-I: YV. Bar-On Emotional Quotient inventory: Youth version. Technical manual. Toronto: Multi Health Systems, Inc.

Barroso-Hurtado, D. y Bembibre, J. (2019). Revisión de los factores de éxito en la promoción de comportamientos prosociales como estrategia preventiva en la justicia juvenil en España. Revista Complutense de Educación, 30(1). https://doi. org/10.5209/rced.55509

Benito, S. C. (2017). Diseño, desarrollo y evaluación del programa "VERA" de educación emocional en la etapa de Educación Primaria (Tesis doctoral, UNED).

Bisquerra, R., Martínez, F., Obiols, M. y Pérez, N. (2006). Evaluación de 360: una aplicación a la educación emocional. Revista de Investigación Educativa, 24(1), 187-203. https://revistas.um.es/rie/article/view/97371

Blewitt, C., Fuller-Tyszkiewicz, M., Nolan, A., Bergmeier, H., Vicary, D., Huang, T., McCabe, P., McKay, T. y Skouteris, H. (2018). Social and emotional learning associated with universal curriculum-based interventions in early childhood education and care centers. A systematic review and meta-analysis. 7AMA Network Open, 1(8), 1-19. https://doi.org/10.1001/jamanetworkopen.2018.5727

Caprara, G. V. y Pastorelli, C. (1993). Early emotional instability, prosocial behaviour, and aggression: Some methodological aspects. European Fournal of Personality, 7(1), 19-36. https://doi.org/10.1002/per.2410070103

Caprara, G. V., Luengo, B. P., Zuffianò, A., Gerbino, M. y Pastorelli, C. (2015). Why and how to promote adolescents' prosocial behaviors: Direct, mediated 
and moderated effects of the CEPIDEA school-based program. Fournal of Youth and Adolescence, 44, 2211-2229. https://doi.org/10.1007/s10964-015-0293-1

Carrillo, G. B. (2016). Validación de un programa lúdico para la mejora de las habilidades sociales en niños de 9 a 12 años (Tesis doctoral, Universidad de Granada).

Cefai, C., Bartolo, P. A., Cavioni, V. y Downes, P. (2018). Strengthening Social and Emotional Education as a core curricular area across the EU. A review of the international evidence. NESET II report. Luxembourg: Publications Office of the European Union. https://doi.org/10.2766/664439

Cejudo, M. J. (2015). Diseño, desarrollo y evaluación del programa "Dulcinea" de educación emocional en un centro de educación secundaria desde la integración de modelos de inteligencia emocional (Tesis doctoral, UNED).

Cohen, L., Manion, L. y Morrison K. (2010). Research methods in education. London: Routlegde.

Consejo de la Unión Europea (2018). Recomendación del Consejo de 22 de mayo de 2018 relativa a las competencias clave para el aprendizaje permanente. Diario Oficial de la Unión Europea, 2018/C, 189, 1-13.

Correa, M. C. (2017). Aproximaciones epistemológicas y conceptuales de la conducta prosocial. Zona próxima, 27, 1-21. https://doi.org/10.14482/zp.27.10978

Coskun, K. (2019). Evaluation of the socio emotional learning (SEL) activities on self- regulation skills among primary school children. The Qualitative Report, 24(4), 764- 780.

Crean, H. F. y Johnson, D. B. (2013). Promoting Alternative Thinking Strategies (PATHS) and elementary school aged children's aggression: Results from a cluster randomized trial. American fournal of Community Psychology, 52(1-2), 56-72. https://doi.org/10.1007/s10464-013-9576-4

Decreto 89/2014, de 1 de agosto, por el que se establece la ordenación y el currículo de la Educación Primaria en la Comunidad Autónoma de Canarias. Boletín Oficial de Canarias, 13 de agosto de 2014, núm. 156, pp. 21911 a 22582. http:// www.gobiernodecanarias.org/boc/2014/156/001.html

del Barrio, M. V., Moreno, C. y López, R. (2001). Evaluación de la agresión e inestabilidad emocional en niños españoles y su relación con la depresión. Clínica y Salud, 13, 33-50. https://journals.copmadrid.org/clysa/art/be83ab3 ecd0db773eb2dc1b0a17836a1

del Moral, M. E. y Villalustre, L. (2018). Análisis de serious games anti-bullying: recursos lúdicos para promover habilidades prosociales en escolares. Revista Complutense de Educación, 29(4). https://doi.org/10.5209/rced.55419

Domitrovich, C. E., Durlak, J. A., Staley, K. C. y Weissberg, R. P. (2017). Socialemotional competence: An essential factor for promoting positive adjustment 
and reducing risk in school children. Child Development, 88(2), 408-416. https://doi.org/10.1111/cdev.12739

Extremera, N., Fernández-Berrocal, P., Mestre, J. M. y Guil, R. (2004). Medidas de evaluación de la inteligencia emocional. Revista Latinoamericana de Psicología, 36(2), 209-228. https://www.academia.edu/19301876/Medidas_de_ evaluaci\%C3\%B3n_de_la_inteligencia_emocional

Ferrándiz, C., Hernández, D., Bermejo, R., Ferrando, M. y Sáinz, M. (2012). Social and emotional intelligence in childhood and adolescence: Spanish validation of a measurement instrument. Revista Psicodidáctica, 17, 309-338. https:// doi.org/10.1387/Rev.Psicodidact.2814

Filella-Guiu, G., Pérez-Escoda, N., Agulló, M. J. y Oriol, X. (2014). Resultados de la aplicación de un programa de educación emocional en Educación Primaria. Estudios sobre Educación, 26, 125-147.

Fuster, D. E. y Altamirano, E. M. (2017). Competencia emocional como elemento fundamental del rendimiento académico de los estudiantes del cuarto y quinto grado de educación primaria. PsiqueMag, 6(1), 257-267.

Goleman, D. (1995). Inteligencia Emocional. Barcelona: Kairós.

Greenberg, M. T., Domitrovich, C. E., Weissberg, R. P. y Durlak, J. A. (2017). Social and emotional learning and education: adopting a public health approach. The Future of Children, 27(1), 13-32.

Greenberg, M., Kusche, C., Cook, E. y Quamma, J. (1995). Promoting emotional competence in school-aged children: the effects of the PATHS curriculum. Development and Psychopathology, 7(1), 117-136. https://doi.org/10.1017/ S0954579400006374

Guo, Q., Sun, P., Cai, M., Zhang, X. y Song, K. (2019). Why are smarter individuals more prosocial? A study on the mediating roles of empathy and moral identity. Intelligence, 75, 1-8. https://doi.org/10.1016/j.intell.2019.02.006

Hortigüela, D., Ausín, V., Abella, V. y Delgado, V. (2017). Percepción de docentes y estudiantes sobre el trabajo de la educación emocional en el aula. Contextos Educativos, 20, 27-41. https://doi.org/10.18172/con.2981

Kusumaningrum, F. A. (2019). Interpersonal intelligence and prosocial behavior among elementary school students. Management Science Letters, 9, 1645-1654. https://doi.org/10.5267/j.msl.2019.5.023

Lemes, N., García, S. y García, J. (2014). Recursos para la Educación Emocional y la Creatividad. Canarias: Conserjería de Educación, Universidades y Sostenibilidad.

Ley 7/2010, de 20 de julio, de Educación de Castilla-La Mancha. Boletín Oficial del Estado, 13 de octubre, núm. 248, pp. 86359 a 86415 . https://www.boe.es/boe/ dias/2010/10/13/pdfs/BOE-A-2010-15624.pdf 
Ley Orgánica 2/2006, de 3 de mayo, de Educación. Boletín Oficial del Estado, 4 de mayo, núm. 106, pp. 17158 a 17207. https://www.boe.es/boe/dias/2006/05/04/ pdfs/A17158-17207.pdf

Ley Orgánica 8/2013, de 9 de diciembre, para la Mejora de la Calidad Educativa. Boletín Oficial del Estado, 10 de diciembre, núm. 295, pp. 97858 a 97921. https://www.boe.es/boe/dias/2013/12/10/pdfs/BOE-A-2013-12886.pdf

Mavroveli, S. y Sánchez-Ruiz, M. (2011). Trait emotional intelligence influences on academic achievement and school behavior. British Fournal of Educational Psychology, 81(1), 112-134. https://doi.org/10.1348/2044-8279.002009

Melero, M. A. y Palomera, R. (2011). Evaluación psicológica del proyecto VyVe. Santander: Fundación Botín.

Modzelewski, H. (2012). La educabilidad de las emociones y su importancia para el desarrollo de un ethos democrático. La teoría de las emociones de Martha Nussbaum y su expansión a través del concepto de autorreflexión (Tesis doctoral, Universidad de Valencia).

Nelsen, J., Lott, L. y Glenn, S. (2008). Disciplina positiva de la A a la Z: 1001 soluciones para los problemas cotidianos en la educación de los hijos. Barcelona: Medici.

Orden ECD/65/2015, de 21 de enero, por la que se describen las relaciones entre las competencias, los contenidos y los criterios de evaluación de la educación primaria, la educación secundaria obligatoria y el bachillerato. Boletín Oficial del Estado, 29 de enero, núm. 25, pp. 6986-7003. http://www.boe.es/boe/ dias/2015/01/29/pdfs/BOE-A-2015-738.pdf

Ortuño-Sierra, J., Fonseca-Pedrero, E., Inchausti, F. y Sastre i Riba, S. (2016). Evaluación de dificultades emocionales y comportamentales en población infanto-juvenil: el cuestionario de capacidades y dificultades (SDQ). Papeles del Psicólogo, 37(1), 14-26. https://www.redalyc.org/jatsRepo/778/77844204003/ index.html

Palomera, R., Melero, M. A. y Briones, E. (2018). Impacto psicológico del programa Educación Responsable. Santander: Fundación Botín.

Panayiotou, M., Humphrey, N. y Wigelsworth, N. (2019). An empirical basis for linking social and emotional learning to academic performance. Contemporary Educational Psychology, 56, 193-204. https://doi.org/10.1016/j.cedpsych.2019.01.009

Parker, J. D., Summerfeldt, L. J., Taylor, R. N., Kloosterman, P. H. y Keefer, K. V. (2013). Problem gambling, gaming and Internet use in adolescents: Relationships with emotional intelligence in clinical and special needs samples. Personality and Individual Differences, 55(3), 288-293. https://doi.org/10.1016/j. paid.2013.02.025 
Pérez Rojo, C. M. (2014). Relación entre inteligencia emocional y conducta prosocial en alumnos de $3^{\circ}$ ciclo de educación primaria. En M. Sánchez Martín, A. B. Mirete Ruiz y N. Orcajada Sánchez (Eds.), Investigación educativa en las aulas de Primaria (pp. 323-336). Murcia: Universidad de Murcia.

Pérez-Escoda, N., Filella, G., Alegre, A. y Bisquerra, R. (2012). Desarrollo de la competencia emocional de maestros y alumnos en contextos escolares. Electronic Fournal of Research in Educational Psycology, 10(3), 1183-1208. https:// www.researchgate.net/publication/259624258_Desarrollo_de_la_competencia_emocional_de_maestros_y_alumnos_en_contextos_escolares

Pérez-Escoda, N., Filella, G., Soldevila, A. y Fondevila, A. (2013). Evaluación de un programa de educación emocional para profesorado de primaria. Educación XX1, 16(1), 233-254. https://doi.org/10.5944/educxx1.16.1.725

Ponce, N. y Aguaded, E. M. (2016). Evaluación de un programa de intervención educativa en inteligencia emocional. Dilemas contemporáneos: Educación, Política y Valores, 2, 1-33. https://www.researchgate.net/publication/317012139_Evaluacion_de_un_programa_de_intervencion_educativa_en_inteligencia_emocional

Pulido, F. y Herrera, F. (2017). La inteligencia emocional como predictora del rendimiento académico en la infancia: El contexto pluricultural de Ceuta. Revista Complutense de Educación, 28(4), 1251-1265. https://doi.org/10.5209/ RCED.51712

Resurrección, D. M., Salguero, J. M. y Ruiz-Aranda, D. (2014). Emotional intelligence and psychological maladjustment in adolescence: A systematic review. Fournal of Adolescence, 37(4), 461-472. https://doi.org/10.1016/j.adolescence.2014.03.012

Reyes, M. R., Brackett, M. A., Rivers, S. E., Elbertson, N. A. y Salovey, P. (2012). The interaction effects of program training, dosage, and implementation quality on targeted student outcomes for the RULER approach to social and emotional learning. School Psychology Review, 41(1), 82-99. https://psycnet.apa. org/record/2012-09813-005

Rodríguez García, G. A. (2018). Eficacia del programa de inteligencia emocional intensivo (PIEI) en la inteligencia emocional y la conducta prosocial de alumnos de la ESO (Tesis doctoral, Universidad Complutense de Madrid).

Ruvalcaba-Romero, N. A., Gallegos-Guajardo, J. y Fuerte, J. M. (2017). Competencias socioemocionales como predictoras de conductas prosociales y clima escolar positivo en adolescentes. Revista Interuniversitaria de Formación del Profesorado, 88(31.1), 77-90.

Tur-Porcar, A., Doménech, A. y Mestre, V. (2018). Vínculos familiares e inclusión 
social. Variables predictoras de la conducta prosocial en la infancia. Anales de Psicología, 34(2), 340-348. https://doi.org/10.6018/analesps.34.2.308151

Tur-Porcar, A., Mestre, V., Samper, P., Malonda, E. y Llorca, A. (2014). Programa de educación de las emociones: la con-vivencia. Evaluación de su eficacia en la conducta prosocial. International fournal of Developmental and Educational Psychology, 1(2), 593-599. http://roderic.uv.es/handle/10550/56825

Vázquez de Prada, C. y López, S. (2016). Resultados de la aplicación de un programa para la mejora de la inteligencia emocional en alumnos de $4^{\circ}$ de Primaria. En J. L. Soler, L. Aparicio, O. Díaz, E. Escolano y M. A. Rodríguez (Coords.), Inteligencia Emocional y Bienestar II. Reflexiones, experiencias profesionales e investigaciones (pp. 807-816). Zaragoza: San Jorge.

Vázquez Verdera, V. (2009). La educación y la ética del cuidado en el pensamiento de Nel Noddings (Tesis doctoral, Universidad de Valencia).

Viguer, P., Cantero, M. J. y Bañuls, R. (2017). Enhancing emotional intelligence at school: Evaluation of the effectiveness of a two-year intervention program in Spanish pre-adolescents. Personality and Individual Differences, 113, 193-200. https://doi.org/10.1016/j.paid.2017.03.036

Weissberg, R. P., Goren, P., Domitrovich, C. y Dusenbury, L. (2013). CASEL guide effective social and emotional learning programs: preschool and elementary school edition. Chicago: CASEL.

Zorrilla, L. (2017). Diseño y aplicación del programa socioemocional Siente Fugando en alumnado de Educación Primaria mediante la metodología Aprendizaje-Servicio (Tesis doctoral, Universitat Jaume I).

Zumbo, B. D., Gadermann, A. M. y Zeisser, C. (2007). Ordinal versions of coefficients alpha and theta for Likert rating scales. Fournal of Modern Applied Statistical Methods, 6(1), 21-29. 
